\section{POS0604 LONG TERM SAFETY AND TOLERABILITY WITH ETANERCEPT BIOSIMILAR (YLB113), RESULTS FROM A 2-YEAR OPEN LABEL EXTENSION STUDY (STUDY NO. YLB113-003)}

Y. Hisashi ${ }^{1}$, Y. Tanaka ${ }^{2}$, T. Hibino ${ }^{3}$, C. Shah ${ }^{4}$, D. Bakhle ${ }^{5}$, D. Stefanidis ${ }^{6} .{ }^{1}$ Sanno Medical Center, Rheumatology, Tokyo, Japan; ${ }^{2}$ University of Occupational and Environmental Health, Japan, Professor, Fukuoka, Japan; ${ }^{3} Y L$ Biologics Ltd, Clinical, Tokyo, Japan ${ }^{4}$ Lupin LTD, Clinical Development, Pune, India; ${ }^{5}$ Lupin LTD, Medical Research, Pune, India; ${ }^{6}$ Viatris GmbH, Medical Affairs, Bad Homburg vor der Höhe, Germany

Background: Eligible subjects with moderate-to-severely active rheumatoid arthritis (RA) who completed a phase 3 double-blind comparative efficacy and safety study (Study No. YLB113-002; Yamanaka et al, 2019) of $50 \mathrm{mg}$ etanercept biosimilar (YLB113) or etanercept reference product (RP) by subcutaneous administration with concomitant MTX treatment for 52 weeks, were enrolled in this open label extension (OLE) study (Study No. YLB113003) to assess the long-term safety and tolerability of YLB113 through to 3 years.

Objectives: This OLE study aimed to evaluate the long-term safety of YLB113 administration in subjects with RA who received RP or YLB113 in a phase 3 active comparator study. The main parameters assessed were safety and tolerability in terms of adverse events (AEs) and injection site reactions (ISRs), incidence of immunogenicity and efficacy as DAS28 improvement (disease activity score in 28 joints)

Methods: Subjects received $50 \mathrm{mg}$ of YLB113 subcutaneously once every 1 to 2 weeks. Safety was assessed by AEs after study drug administration, ISRs, physical examination findings, and immunogenicity. Efficacy (DAS28 score) was assessed at the time of transition to the OLE study (Week 0), and at weeks 12, 24, 48, 72, 96 and at the end of the study. Results: 201 (Full analysis set [FAS]) subjects received the study drug and 184 subjects completed the study (91.5\% completion rate). The average drug exposure in 94 subjects who continued to receive YLB113 and completed the study (94.0\% completion rate) and 90 subjects who switched from RP to YLB113 and completed the study ( $89.1 \%$ completion rate) was 103 weeks providing the longterm drug exposure data of YLB113.

The CTCAE Grade of TEAEs and ADRs observed were Grade 2 or less in severity (with no Grade $\geq 3$ ). The overall incidence of ISRs was $10.0 \%(20 / 201$ subjects) 77 events. All the ISRs reported were Grade 1 except for one Grade 2. An overview of the AEs experienced by the subjects is summarized in the Table 1.

Table 1. Overview of Adverse events in OLE study

\begin{tabular}{lll}
\hline FAS & $\mathbf{n}(\%)$ & Number of events \\
\hline Number of subjects & 201 & - \\
Treatment emergent adverse events (TEAEs) & $188(93.5)$ & 975 \\
Adverse drug reactions (ADRs) & $82(40.8)$ & 221 \\
Serious adverse events (SAEs) & $21(10.4)$ & 27 \\
Serious adverse drug reactions (SADRs) & $7(3.5)$ & 7 \\
AEs leading to premature study discontinuation & $4(2.0)$ & 6 \\
ADRs leading to premature study discontinuation & $3(1.5)$ & 4 \\
SAEs leading to premature study discontinuation & $3(1.5)$ & 4 \\
SADRs leading to premature study discontinuation & $2(1.0)$ & 2 \\
\hline
\end{tabular}

$\mathrm{n}$ - number of subjects with at least $1 \mathrm{AE}$ in the category
The mean DAS28 (mean \pm S.D.) of $2.22 \pm 0.95$ at the study transition was 2.10 \pm 0.91 at Week 72 and $2.06 \pm 0.89$ at the end of the study. It was confirmed that DAS28 slightly decreased with time after the study transition and continued until the end of the study. The average DAS28 value remained low even with longterm administration of YLB113, suggesting that the effects of the study drug was sustained (Figure 1).

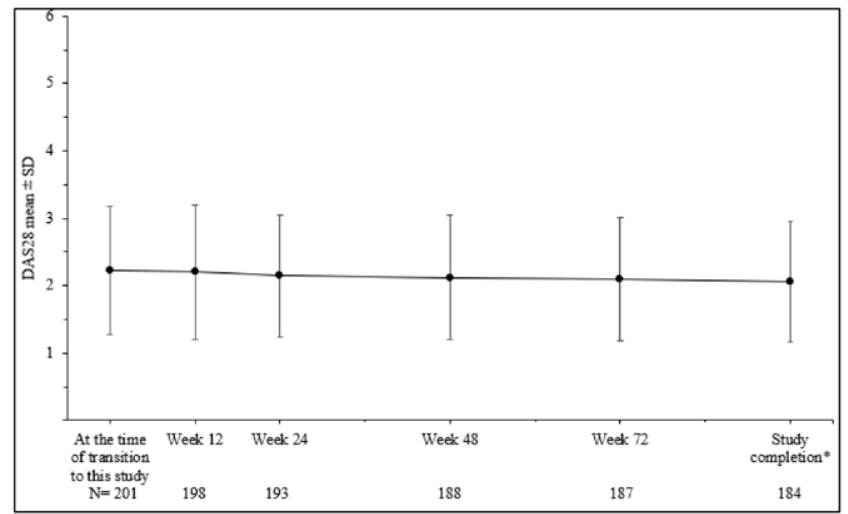

Figure 1. DAS28 over time with YLB113N: Number of subjects. * The administration period at the study completion differs for each subject, because the study transition time differs for each subject.

cts who tested positive for anti-drug antibodies (ADA) at least once in the OLE study were $1.0 \%$ (2/200 subjects). All ADA were transient and disappeared at study completion. Such transient ADA formation was reported in the phase 3 study as well. In the phase 3 study, ADA formation was more frequent in subjects who received RP at 24 weeks, but all subjects were negative in the OLE study after switching. Therefore, switching did not affect immunogenicity.

Conclusion: The safety, efficacy and immunogenicity profile of YLB113 was maintained over the long-term through to 3 years. Switching from RP to YLB113 did not impact safety or immunogenicity.

\section{REFERENCES:}

[1] Yamanaka H, Kamatani N, Tanaka Y, et al. A Comparative Study to Assess the Efficacy, Safety, and Immunogenicity of YLB113 and the Etanercept Reference Product for the Treatment of Patients with Rheumatoid Arthritis. Rheumatol Ther. 2020 Mar;7(1) 149-163.

Disclosure of Interests: Yamanaka Hisashi Speakers bureau: YL Biologics Ltd, Consultant of: YL Biologics Ltd, Yoshiya Tanaka Speakers bureau: Received speaking fees and/or honoraria from Daiichi-Sankyo, Eli Lilly, Novartis, YL Biologics, Bristol-Myers, Eisai, Chugai, Abbvie, Astellas, Pfizer, Sanofi, Asahi-kasei, GSK, Mitsubishi-Tanabe, Gilead, Janssen., Grant/research support from: Received research grants from Abbvie, Mitsubishi-Tanabe, Chugai, Asahi-Kasei, Eisai, Takeda, Daiichi-Sankyo., Toshihiko Hibino: None declared, Chirag Shah Shareholder of: Lupin LTD, Employee of: Lupin LTD, Dhananjay Bakhle Shareholder of: As part of Employee Stock Options Plan of Lupin LTD, Employee of: Lupin LTD, Dimitris Stefanidis Employee of: Sr. Director, Global Medical Affairs Lead, Immunology Biosimilars for Viatris $\mathrm{GmbH}$ DOI: 10.1136/annrheumdis-2021-eular.1030 